\section{P842 HEALTHCARE ENGAGEMENT AND HPV VACCINATION AMONG GAY, BISEXUAL, AND OTHER MEN WHO HAVE SEX WITH MEN (GBMSM): A CIRN STUDY}

${ }^{1}$ Ramandip Grewal* ${ }^{*}{ }^{2}$ Anna Yeung, ${ }^{3}$ Marc Brisson, ${ }^{4}$ Troy Grennan, ${ }^{5}$ Alexandra De Pokomandy, ${ }^{5}$ Joseph Cox, ${ }^{6}$ Gilles Lambert, ${ }^{7}$ David Moore, ${ }^{8}$ François Coutlée, ${ }^{9}$ Shelley Deeks, ${ }^{10}$ Sandra Gardner, ${ }^{11}$ Dane Griffiths, ${ }^{1}$ Wanrudee Isaranuwatchai, ${ }^{12}$ Jody Jollimore, ${ }^{13}$ James Murray, ${ }^{14} \mathrm{Gina}$ Ogilvie, ${ }^{15} \mathrm{Chantal}$ Sauvageau, ${ }^{2}$ Darrell Tan, ${ }^{16}$ Barry Adam, ${ }^{14}$ Heather Armstrong, ${ }^{10}$ Mark Gaspar, ${ }^{17}$ Clemon George, ${ }^{18}$ Daniel Grace, ${ }^{19}$ Trevor Hart, ${ }^{2}$ Ann Burchell. 'St. Michael's Hospital, Toronto, Canada; ${ }^{2}$ St. Michael's Hospital, Centre for Urban Health Solutions, Li Ka Shing Knowledge Institute, Toronto, Canada; ${ }^{3}$ Université Laval, Quebec City, Canada; ${ }^{4} B C$ Centre for Disease Control, Clinical Prevention Services, Vancouver, Canada; ${ }^{5}$ McGill University, Montreal, Canada; ${ }^{6}$ University of Montreal, Montreal, Canada; ${ }^{7} B C$ Centre for Excellence in HIVIAIDS, Vancouver, Canada; ${ }^{8} /$ 'Université de Montréal, Microbiologie Médicale et Infectiologie, Montreal, Canada; ${ }^{9}$ Public Health Ontario, Toronto, Canada; ${ }^{10}$ University of Toronto, Toronto, Canada; ${ }^{11}$ Gay Men's Sexual Health Alliance, Toronto, Canada; ${ }^{12}$ Community Based Research Centre, Vancouver, Canada; ${ }^{13}$ Ontario Ministry of Health and Long-Term Care, Toronto, Canada; ${ }^{14}$ University of British Columbia, Vancouver, Canada; ${ }^{15}$ Institut National de Santé Publique du Québec, Montreal, Canada; ${ }^{16}$ University of Windsor, Windsor, Canada; ${ }^{17}$ University of West Indies - Cave Hill, Cave Hill, Barbados; ${ }^{18}$ University of Toronto, Dalla Lana School of Public Health, Toronto, Canada; ${ }^{19}$ Ryerson University, Psychology, Toronto, Canada

10.1136/sextrans-2019-sti.887

Background Canada was one of the first countries to offer publicly funded programs providing HPV vaccine free of charge to gbMSM. In 2015-2016, the provinces of British Columbia (BC), Ontario (ON), and Quebec (QC) implemented programs for gbMSM aged 9-26 years. We sought to explore where men received the HPV vaccine and the influence of healthcare engagement on vaccination.

Methods Engage is a sexual health study among gbMSM aged $16+$ in the largest urban centres in each province: Vancouver, BC; Toronto, ON; and Montreal, QC. Men are recruited via respondent driven sampling (RDS). We compared proportions (non-RDS adjusted) to questionnaire responses on healthcare engagement among vaccinated $(1+$ doses) versus unvaccinated in the subset of men aged $\leq 26$ years old at enrolment.

Results From 01/2017 to 31/12/2018, 477 men aged $\leq 26$ enrolled (144 Vancouver, 84 Toronto, 249 Montreal). Their median age was 24 years (IQR 22-25). In Vancouver, Toronto and Montreal, respectively, 48.6\%, 44.1\%, 44.2\% had initiated HPV vaccination with $58.6 \%, 56.8 \%$ and $48.2 \%$ of vaccinated men having received all 3 doses. Popular venues for vaccination included a: sexual health clinic $(50.7 \%)$, medical clinic not specializing in sexual health (14.8\%), community health centre (10.6\%), and doctor's office (7.4\%). Compared to unvaccinated men, more vaccinated men had a family doctor $(65.9 \%$ vs $52.3 \%, \mathrm{p}=0.0047)$, had a different provider/clinic for sexual health care or had a HIV care provider $(66.8 \%$ vs $51.8 \%, \mathrm{p}=0.0022)$, had a STI/HIV test in the past year $(90.8 \%$ vs $68.6 \%, \mathrm{p}<0.0001)$, and were diagnosed with a STI in the past year $(39.6 \%$ vs $20.0 \%$, $\mathrm{p}<0.0001)$.

Conclusion Compared to unvaccinated men, vaccinated men were more engaged in healthcare. It is unknown whether men requested the vaccine or providers offered it. Nevertheless, our findings suggest opportunistic HPV vaccination when men receive other services, particularly those related to sexual health.

Disclosure No significant relationships.

\section{P843 \\ THE TRANSMISSION OF HPV IN THE SEXUAL NETWORKS OF SWINGERS, A MULTILEVEL NETWORK APPROACH}

${ }^{1}$ Anne-Marie Niekamp*, ${ }^{2}$ Fleur Koene, ${ }^{3}$ Petra Wolffs, ${ }^{1}$ Christian Hoebe. ${ }^{1}$ Public Health Service South Limburg, Maastricht University Medical Center (MUMC), Sexual Health, Infectious Diseases and Environmental Health, Medical Microbiology, Care and Public Health Research Institute (CAPHRI), Heerlen, Netherlands; ${ }^{2}$ Amsterdam UMC , Medical Microbiology, Amsterdam, Netherlands; ${ }^{3}$ Maastricht University Medical Center (MUMC), Medical Microbiology, Care and Public Health Research Institute (CAPHRI), Maastricht, Netherlands

\subsection{6/sextrans-2019-sti.888}

Background Swingers, i.e. heterosexual couples who, as a couple, have sex with others within the swingers' subculture, are a subpopulation at risk for Sexually Transmitted Infections (STI). Human papillomavirus (HPV) has proven to be highly prevalent in female swingers (unpublished data). The aim of this study is to assess how the different HPV genotypes are distributed within the sexual networks of swingers and whether certain HPV genotypes cluster.

Methods We build a sexual network of swingers based on the network data given by 115 swingers who participated in our prospective cohort network study on swingers between April 2011 and March 2012. The vaginal samples of all female swingers in the network and known in our STI clinic were tested for HPV. HPV genotypes were identified by the highly sensitive $\mathrm{SPF}_{10}-\mathrm{LiPA}_{25}$ method detecting 25 different genital HPV genotypes. The multilevel transmission network consisted of the one-mode sexual network of the swingers and the twomode network of swingers and the HPV genotypes. Distribution and clustering of HPV genotypes in the sexual network was assessed using multilevel network analysis.

Results The sexual network contained 1231 individual swingers of whom 556 female swingers. The sexual network consists of 38 separate components. Of 103 female swingers known in our STI clinic and tested for HPV, 96 (93\%) were positive for at least one of the $25 \mathrm{HPV}$ genotypes. All 25 HPV genotypes were present, but not equally distributed throughout the network. Some genotypes clustered together in specific components of the sexual network.

Conclusion All assessed genotypes of HPV were prevalent in the sexual networks of swingers. Despite the incomplete data in our study, multilevel network analysis turns out to be useful for studying transmission networks. Clustering of highly prevalent HPV could be used as a marker for sexual behaviour, potential STI risk and transmission in swingers.

Disclosure No significant relationships.

\section{P844 THE BURDEN OF HPV AND HSV-RELATED ANOGENITAL DISEASES IN A SOUTHEASTERN US URBAN HIV CLINIC}

${ }^{1}$ Yuanfan Ye*, ${ }^{2}$ Greer Burkholder, ${ }^{1}$ Howard Wiener, ${ }^{1}$ Sadeep Shrestha. ${ }^{1}$ University of Alabama at Birmingham, Epidemiology, Birmingham, USA; ${ }^{2}$ University of Alabama at Birmingham, Medicine, Birmingham, USA

10.1136/sextrans-2019-sti.889

Background Although HPV- and HSV-related conditions (HPVCs, HSVCs) have increased in PLWH, incidence, racial, and gender disparities of the conditions are unclear. We 
examined the public health burden of anogenital HPVCs and HSVCs among PLWH from a southeastern US HIV clinic.

Methods Electronic health records from the HIV Clinic at University of Alabama, Birmingham (01/2006-03/2018) were reviewed. Patients $\geq 18$ years at HIV diagnoses with $\geq 2$ clinical visits were analyzed. Incidence rates of HPVCs (cervical and vaginal/vulvar low and high grade squamous intraepithelial lesion (LSIL, HSIL) and cancers in women, penile cancers in men, warts, anal LSIL, HSIL, and cancers in both) and herpetic ulcers were calculated. Each condition was counted only once at its first diagnosis in the period. We used Joinpoint regression to estimate average annual percentage changes (AAPCs).

Results There were 1038 HPVCs, 546 HSVCs, and 3191 both condition-free, with mean ages: $38.3,39.6$, and 41.3 years, and median nadir CD4 counts: 243, 283, 323 cells $/ \mu \mathrm{L}$, respectively. Incidence of warts, anal LSIL, HSIL, and cancer were different between men $(189,252,44,26$ per 10,000 PYs) and women $(68,15,6,0$ per 10,000 PYs) $(p<0.0001$ for each). Racial disparities were observed in anal LSIL and cancer, cervical HSIL and cancer among whites (rates: 284, 28, 162, 50 per 10,000 PYs) and blacks (rates: 142, 14, 94, 15 per 10,000 PYs), respectively $(p<0.05$ for each). Incident ulcers were higher among women than men (260 vs 163 per 10,000 PYs) and blacks than whites (192 vs 183 per 10,000 PYs). Warts, anal HSIL and cancer, cervical LSIL and cancer increased significantly over time (AAPCs: 19.1, 25.3, 24.9, 13.0, 15.1\%, $\mathrm{P}<0.0001$ for each, respectively).

Conclusion HPVCs and HSVCs are common in the southeastern US PLWH, with substantial increases of warts, anal and cervical lesions and cancers. Better screenings are warranted in the high-risk population.

Disclosure No significant relationships.

\section{P846 BIOMARKERS OF HIV EXPOSURE AND CONDOMLESS RECEPTIVE ANAL SEX IN MEN WHO HAVE SEX WITH MEN USING SELF-COLLECTED RECTAL SWABS}

${ }^{1}$ Maria Lemos, ${ }^{2}$ Vijay Nandi, ${ }^{3}$ Joan Dragavon, ${ }^{1}$ Ira Fleming, ${ }^{1}$ Keertana Krishnan, ${ }^{2}$ Martin Musuruana, ${ }^{1}$ Madeline Kramer, ${ }^{1}$ Hayley Glantz, ${ }^{1}$ Michele Andrasik, ${ }^{4}$ Beryl Koblin, ${ }^{3}$ Robert Coombs, ${ }^{1} \mathrm{M}$ Mcelrath, ${ }^{2}$ Hong Tieu. ${ }^{1}$ Fred Huchinson Cancer Research Center, Vaccine and Infectious Disease Division, Seattle, USA; ${ }^{2}$ Lindsley F. Kimball Research Institute, New York Blood Center, Laboratory of Data Analytics, New York, USA; ${ }^{3}$ University of Washington, Departments of Laboratory Medicine, Seattle, USA; ${ }^{4}$ Lindsley F. Kimball Research Institute, New York Blood Center, Laboratory of Infectious Disease Prevention, New York, USA

10.1136/sextrans-2019-sti.890

Background Biomarkers of HIV exposure could help identify subpopulations at highest risk of HIV acquisition, to focus public health interventions and prevention strategies. This study assessed Y-chromosome single tandem repeat (YSTR) mixtures as biomarkers of receptive anal intercourse without condoms (RAIWC) among men who have sex with men (MSM). We also evaluated the feasibility of self-administered rectal swabs for detection of HIV virions to assess exposures.

Methods Thirty 18-to-50-year-old sexually active, HIV-seronegative MSM were enrolled in New York City. Participants answered daily sexual behavior questions via a mobile phone for 60 days, and were randomized to collecting self-administered rectal swabs daily or after every receptive anal intercourse (RAI) event. Blood collections, rapid HIV diagnostics, and counselling were performed at beginning and end of the study. YSTR mixtures were assessed in DNA from blood and 233 swabs from 20 participants reporting at least one RAIWC event. HIV exposure was measured by virion PCR in 171 swabs linked to reports of RAIWC.

Results As markers of partner's DNA, YSTR mixtures were found in $41 / 138(29.7 \%)$ of self-collected swabs linked to mobile reports of RAI. 15/83 (18\%) swabs collected after reporting abstinence, insertive sex or RAIWC had YSTR mixtures. No participant became HIV infected during the study, yet exposure to HIV was detected in six rectal swabs corresponding to two different participants. For one participant on pre-exposure prophylaxis (PrEP), rectal HIV virions were found after RAIWC with two different HIV positive partners not on antiretroviral therapy. For the second participant, rectal HIV exposures were identified after RAIWC with two different partners disclosing HIV negative status.

Conclusion YSTR mixtures in self-collected rectal swabs demonstrated $82 \%$ specificity but only $30 \%$ sensitivity to assess RAIWC. Detection of HIV exposure in self-collected swabs from two uninfected participant indicates it was possible to measure rectal HIV exposures in MSM.

Disclosure No significant relationships.

\section{P848 REVISITING ENFUVIRTIDE'S MECHANISM AND DESIGNING ITS ANALOG WITH IMPROVED ACTIVITY BY TARGETING TRIPLE SITES IN GP41}

${ }^{1}$ Shibo Jiang ${ }^{*},{ }^{1}$ Wei Xu, ${ }^{2}$ Jing Pu, ${ }^{2}$ Shan Su, ${ }^{2}$ Chen Hua, ${ }^{2}$ Xiaojie Su, ${ }^{2}$ Qian Wang, ${ }^{2}$ Lu Lu. ${ }^{1}$ Fudan University, School of Basic Medical Sciences, Shanghai, China; ${ }^{2}$ Shanghai Medical College of Fudan University, Shanghai, China

10.1136/sextrans-2019-sti.891

Background In the early of 1990's, several peptides overlapping gp41 CHR region, including SJ-2176, T20, and C34 were reported to have potent HIV-1 fusion inhibitory activity. T20 (generic name: enfuvirtide) was approved by the U.S. FDA as the first HIV-1 fusion inhibitory peptide-based antiHIV drug. However, its clinical application is limited because of its low potency and low genetic barrier to resistance. Furthermore, its mechanism of action is still elusive. Therefore, it is essential to define T20's mechanism of action, based on which a new analogous peptide with improved antiviral activity can be designed.

Methods The inhibitory activity of peptides on $6 \mathrm{HB}$ formation was tested in a temperature-controlled cell-cell fusion assay by flow cytometry using 6HB-specific mAb 2G8; on HIV-1 infection and fusion was assessed by p24 and cell-cell fusion assays. Interaction between different peptides or peptide and antibody was evaluated by ELISA.

Results T20 could inhibit 6HB formation at early, but not late, stage of HIV-1 fusion, while T1144 was effective at both stages. T20-SF is much more effective than T20 in binding to FP-P and inhibiting infection of HIV-1, including T20-resistant strains, and FP-P-mediated hemolysis.

Conclusion In the early of 1990's, several peptides overlapping gp41 CHR region, including SJ-2176 (residues 630-659), T20 (residues 638-673), and C34 (residues 628-661) were reported to have potent HIV-1 fusion inhibitory activity. T20 (generic name: enfuvirtide) was approved by the U.S. FDA as 